\title{
Induced fit or conformational selection for RNA/U1A folding
}

\author{
FANG QIN, ${ }^{1}$ YUE CHEN, ${ }^{1}$ MAOYING WU, ${ }^{1}$ YIXUE $\mathrm{LI}^{2}{ }^{2}$ JIAN ZHANG, ${ }^{3}$ and HAI-FENG CHEN ${ }^{1,2}$ \\ ${ }^{1}$ College of Life Sciences and Biotechnology, Shanghai Jiaotong University, Shanghai, 200240, China \\ ${ }^{2}$ Shanghai Center for Bioinformation Technology, Shanghai, 200235, China \\ ${ }^{3}$ Institute of Medical Science, School of Medicine, Shanghai Jiaotong University, Shanghai, 200025, China
}

\begin{abstract}
The hairpin II of U1 snRNA can bind U1A protein with high affinity and specificity. NMR spectra suggest that the loop region of apo-RNA is largely unstructured and undergoes a transition from unstructured to well-folded upon U1Abinding. However, the mechanism that RNA folding coupled protein binding is poorly understood. To get an insight into the mechanism, we have performed explicit-solvent molecular dynamics (MD) to study the folding kinetics of bound RNA and apo-RNA. Roomtemperature MD simulations suggest that the conformation of bound RNA has significant adjustment and becomes more stable upon U1A binding. Kinetic analysis of high-temperature MD simulations shows that bound RNA and apo-RNA unfold via a twostate process, respectively. Both kinetics and free energy landscape analyses indicate that bound RNA folds in the order of RNA contracting, U1A binding, and tertiary folding. The predicted $\Phi$-values suggest that A8, C10, A11, and G16 are key bases for bound RNA folding. Mutant Arg52GIn analysis shows that electrostatic interaction and hydrogen bonds between RNA and U1A (Arg52Gln) decrease. These results are in qualitative agreement with experiments. Furthermore, this method could be used in other studies about biomolecule folding upon receptor binding.
\end{abstract}

Keywords: mRNA splicing complex; RNA folding; binding; transition state; $\Phi$-values

\section{INTRODUCTION}

The U1A protein is a component of the U1 small nuclear ribonucleoprotein ( $n$ RNP), which is one of five large RNAprotein complexes involved in most eukaryotic pre-mRNA splicing (Lührmann et al. 1990; Green 1991). It contains two RNA recognition motifs (RRMs) (Lu and Hall 1995). The $\mathrm{N}$-terminal domain of U1A binds to hairpin II of U1 snRNA and two adjacent internal loops in the $3^{\prime}$ UTR with high affinity and specificity. The AUUGCAC of the conserved 7-nucleotide (nt) sequence of snRNA is the U1A protein binding site (Scherly et al. 1989; van Gelder et al. 1993).

The crystal structure of U1A and snRNA was released in 1994 (pdb code:1URN) (Oubridge et al. 1994). U1A is comprised of a $\beta \alpha \beta \beta \alpha \beta$ sandwich fold that forms a fourstranded $\beta$-sheet flanked on one side by two $\alpha$-helices. The

Reprint requests to: Jian Zhang, Institute of Medical Science, School of Medicine, Shanghai Jiaotong University, 280 Chongqing Road, Shanghai, 200025, China; e-mail: jian.zhang@sjtu.edu.cn; fax: 86-21-34204348; or Hai-Feng Chen, College of Life Sciences and Biotechnology, Shanghai Jiaotong University, 800 Dongchuan Road, Shanghai, 200240, China; e-mail: haifengchen@sjtu.edu.cn.

Article published online ahead of print. Article and publication date are at http://www.rnajournal.org/cgi/doi/10.1261/rna.2008110. 10-nt loop of the RNA hairpin binds to the protein as an open structure. The AUUGCAC sequence of the RNA loop fits into the groove between the $\beta 2-\beta 3$ loop and the $\mathrm{C}$-terminal domain of protein. The last three nucleotides (UCC) in the loop of RNA have no apparent contact with the $\beta$-sheet (see Fig. 1A).

NMR experiments suggest that the chemical shifts of many protons for bound RNA are substantially different from those for apo-RNA, especially in the loop region of the RNA hairpin (Hall 1994). Furthermore, the hairpin loop of apo-RNA is largely unstructured (Oubridge et al. 1994). Then, RNA undergoes a transition from unstructured to well-folded upon U1A binding. However, the mechanism of RNA folding coupled binding is poorly understood. Fortunately, molecular dynamics (MD) simulation is a powerful tool for analyzing the structural and dynamic features of biomacromolecules. Several MD simulations of apo-RNA, apo-U1A, or their complex have been made to reveal the recognition mechanism and the conformational change (Reyes and Kollman 1999; Tang and Nilsson 1999; Blakaj et al. 2001; Pitici et al. 2002; Showalter and Hall 2005; Kormos et al. 2006, 2007; Zhao et al. 2006). The previous works suggest that the structural adaption of 


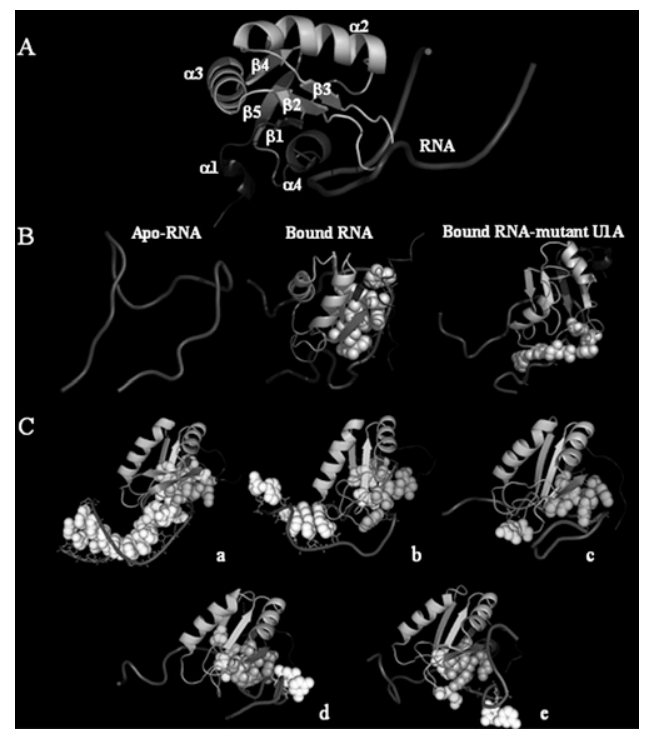

FIGURE 1. The structures of bound and apo states. (A) Ribbon representation of crystal structure for RNA-U1A (pdb code: 1URN). The location of main secondary structures is indicated. $(B)$ The average structures of TSE for apo-RNA, bound RNA, and bound RNA-U1A (Arg52Gln). (C) Unfolding pathway of bound RNA. (a) $<0$ ns (F), (b) $1.38 \mathrm{~ns}(\tau \mathrm{Qf}),(\mathrm{c}) 9.49 \mathrm{~ns}(\tau \mathrm{Qb}),(\mathrm{d}) 11.78 \mathrm{~ns}(\tau \mathrm{Rg}),(\mathrm{e})>15 \mathrm{~ns}(\mathrm{U})$.

RNA and U1A obeys the induced fit mechanism (Koshland 1958; Tang and Nilsson 1999; Pitici et al. 2002; Showalter and Hall 2005). Here we focus on the RNA folding kinetics upon U1A binding. Our research intends to reveal a series of interesting questions: (1) How does the conformation of the RNA hairpin change upon U1A binding? (2) What is the difference in the folding pathway between bound RNA and apo-RNA? (3) Which mechanism does bound RNA folding obey? To shed light on these questions, we utilize atomic molecular dynamics simulation in explicit solvent to analyze the coupling mechanism between RNA folding and U1A binding (Onoa and Tinoco 2004).

However, all atomic MD simulations are currently restricted to the time scale of less than $1 \mu \mathrm{s}$, which is much shorter than the folding half-time of most biomacromolecules at room temperature (at least $1 \mathrm{~ms}$ ) (Baker 1998; Fersht and Daggett 2002). Fortunately, the rate of unfolding can accelerate by approximately six orders of magnitude at high temperature (usually $498 \mathrm{~K}$ ) (Shea and Brooks 2001), so most biomacromolecules unfold in the nanoscale time scale at this temperature (Fersht and Daggett 2002). Furthermore, experiments confirm that the transition state for folding and unfolding is expected to be the same from the principle of microscopic reversibility (Fersht and Daggett 2002). Based on these previous works, unfolding simulations at high temperature have been used in the current study.

In this work, we will discuss the folded states, the unfolding kinetics, the unfolding landscape, transition states, and the unfolding pathway for both bound RNA and apoRNA to understand RNA folding coupled U1A binding.

\section{RESULTS}

\section{Folded state}

Previous work suggests that a small number of trajectories for MD simulation (5-10) are sufficient to capture the average properties of the protein (Day and Daggett 2005). Therefore, 10 trajectories of $10.0 \mathrm{~ns}$ each were simulated at $298 \mathrm{~K}$ to analyze the folded state of apo-RNA, apo-U1A, and their complex. To show the influence of wild-type (WT) and mutant U1A on the stability of the folded RNA, $\mathrm{C}^{\prime}$ and $\mathrm{C} \alpha$ variations for bound and apo states are illustrated in Figure 2. The C5' variations of bound RNA for wild type and mutant are significantly smaller than that of apo-RNA, especially in the hairpin-loop region. The $\mathrm{C} \alpha$ variations of bound U1A for wild type and mutant are also smaller than that of apo-U1A, especially in the loop region between $\beta 2$ and $\beta 3$ fitting into the RNA hairpin. This suggests that both bound RNA and U1A become less flexible and more stable upon binding, which is consistent with previous experiments (Hall 1994; Oubridge et al. 1994). In general, the angle parameters $(\alpha, \beta, \gamma, \delta$, $\varepsilon$, and $\zeta)$ are used to describe the secondary structure of RNA (Murray et al. 2003). Surprisingly, the fluctuations of $\alpha, \beta, \gamma, \delta, \varepsilon$, and $\zeta$ for bound RNA seem larger than that of apo-RNA (shown in Fig. 3). This suggests that the secondary structure of bound RNA changes disorders upon U1A binding. However, the $\Phi / \psi$ variation of bound U1A is similar to that of apo-U1A except in the loop between $\beta 2$ and $\beta 3$ and the C-terminal domain (shown in Supplemental Fig. 1S).

The sugar conformations of bound RNA and apo-RNA are illustrated in Figure 4. Our simulation suggests that the conformation of bases $\mathrm{U} 8$ and $\mathrm{C} 10$ is $\mathrm{C}^{\prime}$-endo in the complex during a 10-ns simulation. This is consistent with experimental observation (Oubridge et al. 1994). Besides, the sugar conformations of bases $\mathrm{C} 12$ and U13 change to C3'-endo of bound RNA from C2'-endo of apo-RNA. This testifies that the sugar conformation of RNA also becomes more stable upon U1A binding.

The landscape of the distance difference for base pairs between bound RNA and apo-RNA is shown in Figure 5A. The landscape can reflect the relative conformational change of the RNA backbone. The deep gray area indicates that the distance difference for bases $6-8$ and $12-14$ is

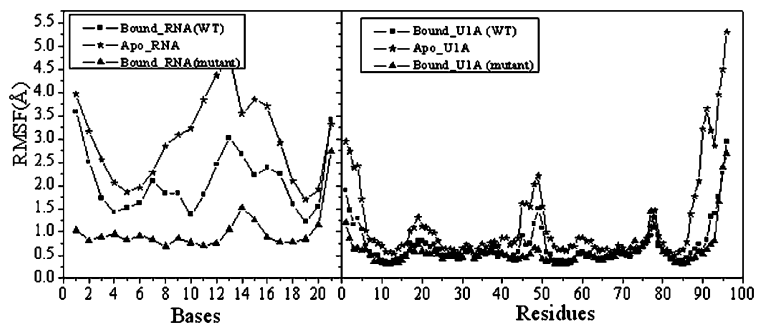

FIGURE 2. $\mathrm{C}^{\prime}$ ' or $\mathrm{C} \alpha$ variation of bound and apo states. 


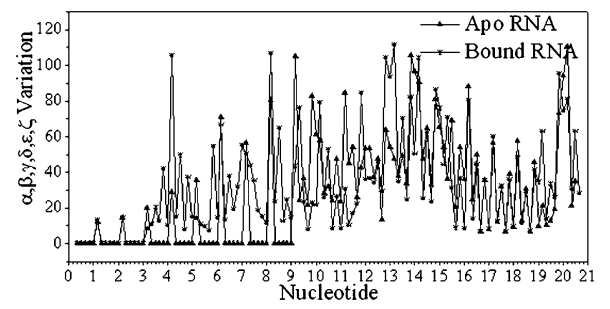

FIGURE 3. $\alpha, \beta, \gamma, \delta$, $\varepsilon$, and $\zeta$ fluctuation for bound RNA and apoRNA.

a positive value. This suggests that nucleotides $12-14$ are stretched upon U1A binding. The French gray area represents that the distance difference is a negative value. This suggests that the stem region of bound RNA bends closer to the loop region, which is consistent with the alignment result of the average structure between bound RNA and apo-RNA (shown in Supplemental Fig. 2S). The distance landscape for residue pairs between bound and apo-U1A is shown in Figure 5B. The result shows that most regions of the U1A backbone did not undergo an obvious conformational change except in the $\beta 2-\beta 3$ loop and C-terminal region. The $\beta 2-\beta 3$ loop and $\mathrm{C}$-terminal region might play key roles in the formation of RNA/U1A complex. This is consistent with experimental observation that the C-terminal region participates in base recognition by forming direct and water-mediated hydrogen bonds (Oubridge et al. 1994).

To study the drive force of the binding-induced conformer change in the folded state, the electrostatic, hydrophobic, and hydrogen-binding interactions between RNA and U1A were analyzed. The electrostatic interactions in the simulation of 10 trajectories are shown in Supplemental Figure 3S. There are 19 electrostatic interactions between positively charged amino acids and the phosphates of RNA, with populations higher than $60 \%$. The positively charged residues, such as Lys20, Lys22, Lys23, Arg47, Lys50, Arg52, Arg83, Lys88, and Lys96, provide electrostatic interactions with the phosphates of the RNA stem and loop regions. Note, also, these positively charged residues are almost around the RNA (shown in Supplemental Fig. 4S). This suggests that the electrostatic interactions between the positively charged amino acids and the negative phosphates of the RNA are so important in the complex formation and its stability. Our simulations are consistent with the mutant experiment of Lys20, Lys22, and Lys23, in which substitutions of these residues resulted in a loss of RNAbinding affinity (Nagai et al. 1990; Law et al. 2006). The hydrophobic contacts in the simulation of 10 trajectories are also shown in Supplemental Figure 3S. Eight stable hydrophobic interactions can be found: C10/Try13, A11/Phe56, C10/Phe56, G16/ Leu49, C12/Leu44, C10/Ala87, A11/Leu44, and U7/Leu49, with populations higher than $60 \%$. These hydrophobic residues of Try13, Leu49, Phe56, and Ala87 are located on the antiparallel four-stranded $\beta$-sheet. Furthermore, U7 and G16 interact with Leu49 on the $\beta 2-\beta 3$ protein loop. This suggests that the RNA hairpin seems to clamp the protein loop. Supplemental Figure 3S also shows 13 hydrogen bonds with populations higher than $60 \%$. These strong hydrogen bonds are between residues Asp92, Ser91, Asp90, Thr89, Lys88, Try86, Glu85, Arg52, and Asn16 and the Watson-Crick edges of A6, G9, C10, A11, C12, and G16. Remarkably, nearly all of the hydrophobic contacts and hydrogen bonds are relative to G9, C10, A11, C12, and G16 of the RNA hairpin, which suggests that these five nucleotides play key roles in RNA conformational adjustment. In summary, U1A binding introduced more electrostatic interactions, hydrophobic contacts, and hydrogen bonds at the interface, and these are responsible for the higher stability in bound RNA.

\section{Unfolding kinetics}

The fraction of native tertiary contacts (Qf) and native binding contacts $(\mathrm{Qb})$ is used to monitor unfolding and unbinding kinetics, respectively. Time evolutions of $\mathrm{Qb}$ and Qf for bound RNA are shown in Figure 6. Figure 6 suggests that the tertiary unfolding and unbinding kinetics can be represented well by single exponential functions, indicating first-order kinetics in the NVT ensemble at $498 \mathrm{~K}$. The fitted kinetics data are listed in Table 1. The kinetics analysis shows that the unbinding half-time is $9.49 \pm 1.03 \mathrm{~ns}$ and the tertiary unfolding half-time is $1.38 \pm 0.038 \mathrm{~ns}$. This indicates that the tertiary unfolding is much faster than the unbinding for bound RNA. The time evolution of Qf for apo-RNA is shown in Figure 7. It is found that the tertiary unfolding of apo-RNA also obeys first-order kinetics, with a half-time of $1.10 \pm 0.034 \mathrm{~ns}$, which is faster than the tertiary unfolding
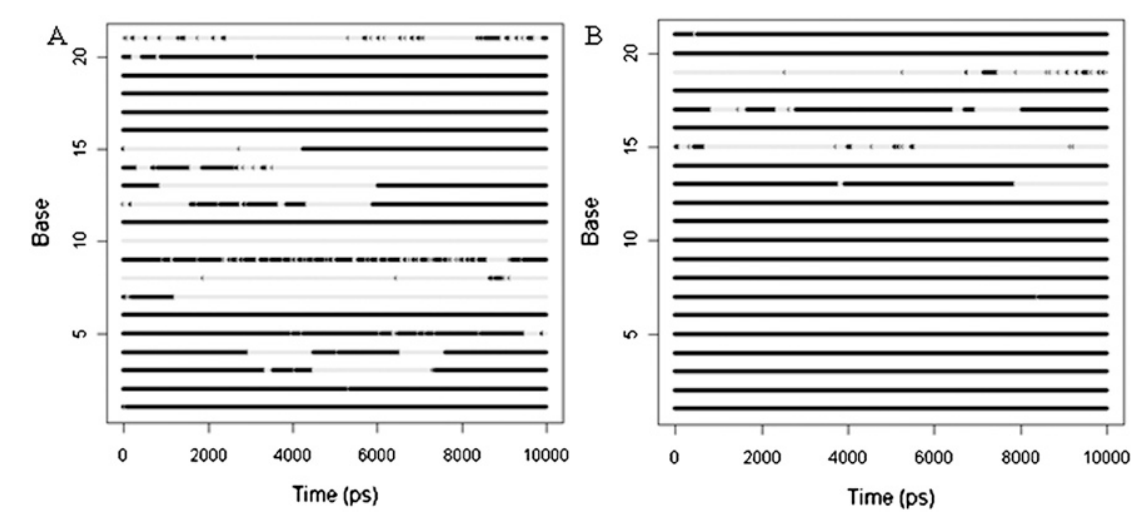

FIGURE 4. The sugar pucker of bound RNA and apo-RNA (black: $\mathrm{C} 3$ '-endo, gray: $\mathrm{C} 2$ 'endo). (A) bound RNA; (B) apo-RNA. 
A

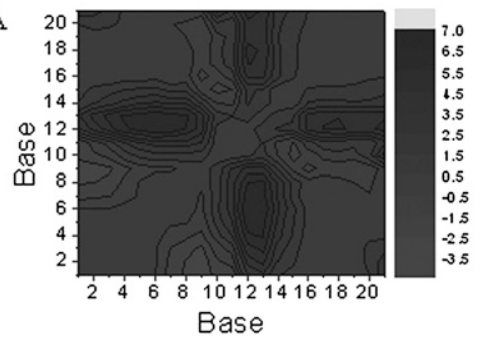

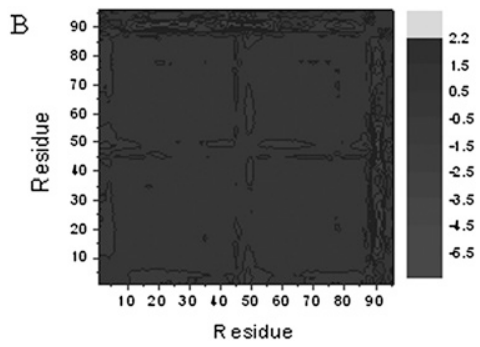

FIGURE 5. The landscape of distance difference between bound and apo state. (A) RNA; $(B)$ U1A.

and unbinding of bound RNA. The time evolution of the radius of gyration $(\mathrm{Rg})$ for bound RNA is also presented in Figure 6. The extension of bound RNA obeys first-order kinetics, and the half-time of $\mathrm{Rg}$ is $11.77 \pm 2.92$ ns. This indicates that the extension of bound RNA is slower than the tertiary unfolding and unbinding. The time evolution of the radius of gyration $(\mathrm{Rg})$ for apo-RNA is shown in Figure 8 . The half-time of $\mathrm{Rg}$ is $2.94 \pm 0.33 \mathrm{~ns}$. This suggests that the tertiary unfolding and extension of bound RNA are postponed upon U1A binding.

\section{Unfolding landscape}

To further understand the interdependence between the RNA folding and U1A binding, the unfolding landscape of bound RNA was analyzed with the variables $\mathrm{Qf}$ and $\mathrm{Qb}$, as shown in Figure 8. The unfolding landscape shows that the tertiary unfolding proceeds first, while the binding contacts are held stable, and then is followed by the unbinding. This is in agreement with the unfolding kinetics analysis for bound RNA and suggests the formation of the tertiary contact depends on the formation of the binding interface.

The coupling between the tertiary unfolding and the extension of RNA is also investigated for both bound (shown in Fig. 8) and apo states (shown in Supplemental Fig. 5S). It was found that Qf decreases first, while the radius of gyration does not change, and then it is followed by the extension of RNA for both systems. This suggests that the extension of RNA is followed by the tertiary unfolding, and this is also consistent with the unfolding kinetics analysis.

\section{Transition state}

Kinetics analysis shows that the tertiary unfolding of bound RNA and apo-RNA obeys first-order kinetics. The tertiary unfolding of bound RNA-mutant U1A (Arg52Gln) also obeys first-order kinetics (shown in Supplemental Fig. 6S). This suggests that bound RNA, apo-RNA, and bound RNAmutant U1A (Arg52Gln) unfold via a two-state process. This is consistent with previous results indicating that mechanical unfolding of the RNA hairpin is stochastic and obeys twostate dynamics (Hyeon and Thirumalai 2005). However, an intermediate state was found during the folding pathway of the RNA hairpin (Bowman et al. 2008). In this simulation, there was a transition state corresponding to the free energy maximum along the unfolding pathway. The structures of the free energy maxima comprise the transition state ensemble (TSE). TSE structures can either fold or unfold, and the transition probability $(P)$ will be $50 \%$. We have scanned MD snapshots for TSE structures in all unfolding trajectories for each of bound RNA, apo-RNA, and bound RNA-mutant U1A(Arg52Gln) (Pande and Rokhsar 1999). The transition probability curves were further fitted by the Boltzmann equation and are shown in Supplemental Figure 7S. The analysis yields 73 snapshots for the bound RNA, 113 snapshots for the apo-RNA, and 13 snapshots for the RNAmutant U1A(Arg52Gln) TSE.

The average structures of bound RNA, apo-RNA, and bound RNA-U1A(Arg52Gln) TSE are shown in Figure 1B. There are five native contacts (G9/Asn15, C10/Tyr13, C10/ Phe56, C10/Ala87, and A11/Phe56) for bound RNA TSE, while there is no native contact for apo-RNA TSE. For the TSE of bound RNA-mutant U1A (Arg52Gln), there are just two native contacts (U7/Glu19 and U8/Asn16). Apparently, it can be concluded that the TSE of bound RNA is more native-like than that of bound RNA-mutant U1A (Arg52Gln) and apo-RNA. This indicates that the relative folding activation free energy of bound $\operatorname{RNA}\left(\Delta G_{\text {bound }}\right)$ is probablly lower than that of apo-RNA $\left(\Delta G_{\text {apo }}\right)$, leading to a relatively faster folding rate for bound RNA. This is consistent with the result that the tertiary unfolding time scale of bound RNA is larger than that of apo-RNA. Furthermore, the TSE of the bound RNA is different from that observed for the bound RNA-mutant U1A. This suggests that the mutation of U1A (Arg52Gln) significantly reduces the RNA affinity.

\section{$\Phi$-Value prediction}

$\Phi$-Values have been widely used to determine key residues in protein folding by theoretical and experimental investigations

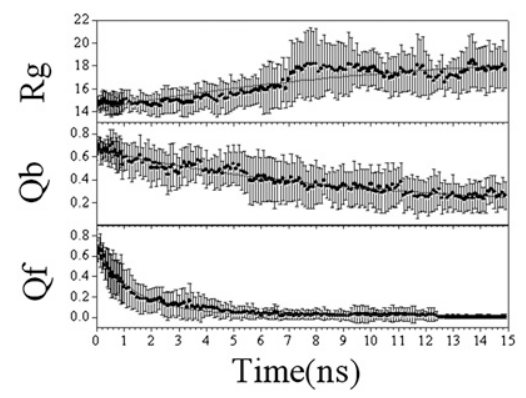

FIGURE 6. Kinetics fitting for bound RNA. 
TABLE 1. Unfolding kinetics constants for bound RNA and apo-RNA

\begin{tabular}{llccccc}
\hline Compound & Property & \multicolumn{2}{c}{$\tau$} \\
& $(\mathrm{ns})$ & $A$ & $B$ & $R^{2}$ \\
\hline Bound RNA & Qb & $9.49 \pm 1.03$ & $0.52 \pm 0.026$ & $0.14 \pm 0.029$ & 0.94 \\
& Qf & $1.38 \pm 0.038$ & $0.64 \pm 0.009$ & $0.020 \pm 0.002$ & 0.98 \\
& Rg & $11.77 \pm 2.92$ & $-5.30 \pm 0.69$ & $19.54 \pm 0.76$ & 0.81 \\
Apo-RNA & Qf & $1.10 \pm 0.034$ & $0.83 \pm 0.012$ & $0.0010 \pm 0.004$ & 0.99 \\
& Rg & $2.94 \pm 0.33$ & $-9.08 \pm 0.33$ & $21.98 \pm 0.37$ & 0.92 \\
\hline
\end{tabular}

Note: The curves are fitted by $A \times \exp (-t / \tau)+B$.

\section{Comparison with other simulations}

The landscape of distance difference between bound RNA and apo-RNA suggests that RNA undergoes a significant conformational change upon U1A binding, especially in the loop region. This is consistent with the observations of the previous simulations (Tang and Nilsson 1999; Pitici et al. 2002; Showalter and Hall 2005).

Our simulation indicates that A8,

(Fersht et al. 1992; Fersht 2000; Fernandez-Escamilla et al. 2004; Sato and Fersht 2007). In this study, all of TSE structures were used to predict $\Phi$-values for bound RNA and apo-RNA. The $\Phi$-values are shown in Figure 9. In general, the $\Phi$-values of bound RNA are significantly larger than those of apo-RNA. This suggests that there are more key bases in RNA folding upon U1A binding. Note also that the highest $\Phi$-values are found for U8, C10, A11, and G16, suggesting that these bases play a key role in the folding of bound RNA. This is consistent with the structural analysis that the critical roles of U8, C10, A11, and G16 form electrostatic interactions, hydrophobic contacts, and hydrogen-bonding networks with U1A. These predicted $\Phi$-values can be confirmed by experiment.

\section{DISCUSSION}

\section{Comparison with experiment}

The structural analysis suggests that Arg52 of U1A is the critical residue in stabilizing the complex (Oubridge et al. 1994). Our room-temperature simulation finds two stable electrostatic interactions for G16/Arg52 and C15/Arg52. Besides electrostatic interactions, there are also three stable hydrogen bonds for A6/Arg52, G9/Arg52, and G16/Arg52. The simulation of mutating Arg52 to Gln suggests that this mutant completely abolishes these stable hydrogen bonds with RNA bases and electrostatic interactions (shown in Supplemental Fig. 8S). This is in agreement with the mutational experiment in which Arg52Gln completely abolishes RNA binding (Nagai et al. 1990).

Furthermore, an X-ray experiment observed the hydrogen-bonding network between RNA and U1A. Hydrogen bonds, such as G9/Asn16, G9/Asn15, U8/Arg93, U8/Lys80, C10/Gln85, C10/Lys88, A11/Ser91, C12/Asp90, Thr89, and C12/Asp92, are also found in our simulation. Besides, there are also two marginally stable hydrogen bonds (U7/Glu19 and U8/Asn16) with a population around 50\%. This is consistent with the observation of the X-ray experiment (Oubridge et al. 1994).

Finally, we predict $\Phi$-values of RNA (shown in Fig. 9) and find that the $\Phi$-values of U8, C10, A11, and G16 are higher than those of other residues for bound RNA. These results are also consistent with previous reports and can be confirmed by experiment.
C10, A11, and G16 are key bases for U1A binding and U13, C14, and C15 have no contact with U1A. This is consistent with the investigation of Tang and Nilsson (1999). Tang and Nilsson (1999). have mentioned that the sugar conformation of U7 changes from 3 '-endo to 2 'endo during the simulation. This could be connected to the high mobility of U8, where purine keeps the $\mathrm{C}^{\prime}$ '-endo pucker and pyrimidine prefers the $\mathrm{C} 2$ '-endo pucker. Those are in accord with our results regarding the sugar conformational adjustment of U7.

Among the 19 stable electrostatic interactions, there were six between Lys20, Lys22, and Lys23 and the phosphates of RNA. This suggests that these positively charged residues are important for complex stability. This is in agreement with previous work showing that electrostatic interactions are important (Hermann and Westhof 1999; Law et al. 2006). In addition to electrostatic interactions, we also found two important hydrophobic contacts (A11/Phe56 and $\mathrm{C10/Phe56).} \mathrm{This} \mathrm{is} \mathrm{consistent} \mathrm{with} \mathrm{the} \mathrm{simulation} \mathrm{in}$ which the Phe56Ala mutant decreased the affinity of RNA (Blakaj et al. 2001).

\section{Convergence and sampling}

Ten trajectories were simulated for bound RNA, apo-RNA, and apo-U1A. First, in this study, we intend to reveal if multiple trajectories are necessary. The populations of eight hydrophobic contacts and 13 hydrogen bonds in 10 trajectories are shown in Supplemental Figures 9S and 10S. The populations of three former hydrophobic contacts and seven hydrogen bonds are very similar among the 10 trajectories.

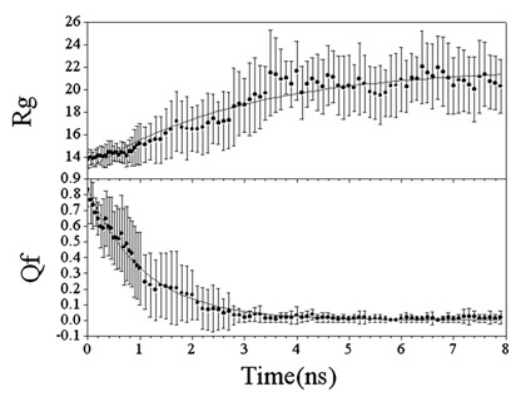

FIGURE 7. Kinetics fitting for apo-RNA. 


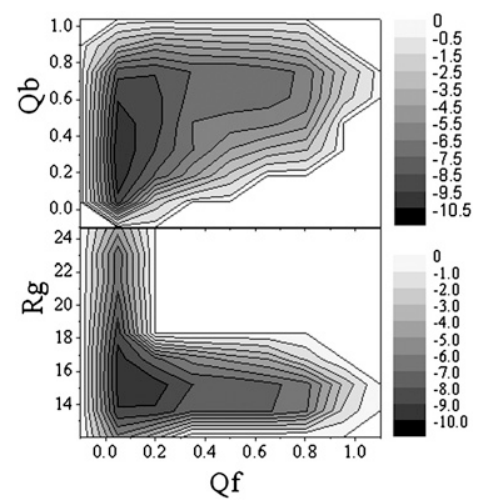

FIGURE 8. Unfolding landscapes with respect to Qb, Rg, and Qf for bound RNA.

However, the remainders have large fluctuations. If we just sample one or two simulations, some stable hydrophobic contacts and hydrogen bonds will be missed. Therefore, multiple simulations are necessary for this system. Second, we will check if 10 trajectories are sufficient for these systems. The average number of hydrophobic contacts and hydrogen bonds with standard error versus the number of trajectories is plotted in Supplemental Figure 11S. As the number of trajectories increases, the number of the hydrophobic contacts and hydrogen bonds with the standard error gradually changes. Finally, the average number and standard error almost remain constant. This is consistent with a previous report in which a small number of MD simulations (5-10) are sufficient to capture the average properties of a protein observed in experiment (Day and Daggett 2005).

\section{Unfolding and folding pathways}

Based on the unfolding kinetics and the unfolding landscape, the unfolding pathway for bound RNA can now be constructed, and it is shown in Figure 1C. (1) At the tertiary unfolding half-time, there are four out of 15 (folded state) native contacts within RNA. Most of the lost native contacts are within the stem region. The native binding contacts between RNA and U1A also start to disappear; still 11 out of 16 exist. (2) At the half-time of U1A unbinding, all of the native contacts within RNA have disappeared. There are four out of 16 native binding contacts remaining. The average time for visiting the transition state is between the half-time of the tertiary unfolding and the unbinding. (3) At the half-time of the extension for RNA, there are one out of 15 native contacts within RNA and five out of 16 between RNA and U1A. (4) For the unfolded state, there are 1 native contact within RNA and 5 binding contacts between RNA and U1A remaining. Interestingly, three native hydrophobic contacts of G9/Asn15, C10/Tyr13, and C10/ Lys88 (see Fig. 1C, gray area) always exist during the unfolding of bound RNA. Furthermore, there are also two native contacts of G9/Asn 15 and C10/Tyr13 included in the
TSE of bound RNA. These base/residue pairs might be nuclei and play key roles in the folding of bound RNA. This is consistent with experimental observation (Oubridge et al. 1994).

Because the unfolding pathways of chymotrypsin inhibitor 2 and the engrailed homeodomain are confirmed to be the reverse of the folding ones (McCully et al. 2008; Day and Daggett 2007), we assume that the folding pathway of RNA also obeys the same rule. Therefore, the proposed folding/binding pathway of bound RNA is RNA contracting, U1A binding, and the tertiary folding. This suggests that U1A binding indeed induces the folding of RNA. The difference between the folding pathways of bound RNA and apo-RNA is the accelerated tertiary folding for RNA upon U1A binding. This is similar to the folding of the TIS11d/ RNA system (Qin et al. 2009).

\section{Induced fit or conformational selection}

Two main models are used to explain the biomolecule folding coupled receptor binding (Boehr and Wright 2008). One is the "induced-fit" model (Koshland 1958), the other is "conformational selection" (Ma et al. 1999, 2002; Tsai et al. 1999, 2001; Kumar et al. 2000; Weikl and von Deuster 2009). If the bound conformation of the biomolecule exists prior to the receptor binding, the receptor will directly select bound conformation, otherwise it will adjust the biomolecule conformation before binding (Turjanski et al. 2008). Recently, a residual dipolar coupling experiment suggested that the folding of the ubiquitin complex obeys conformational selection, rather than the induced-fit mechanism (Lange et al. 2008). Nevertheless, the kinetics character for both mechanisms has also been observed in the same system (James and Tawfik 2003; Okazaki and Takada 2008). In this system, the folding pathway of bound RNA shows that U1A binding is prior to RNA folding. Furthermore, the average structure of the transition state ensemble includes two native binding contacts. These native contacts are favored in the formation of bound RNA conformation. Finally, the conformational clusters based on C $\alpha$ RMSD, relative to the average structures of bound RNA and apoRNA, are illustrated in Figure 10. In the current simulation,

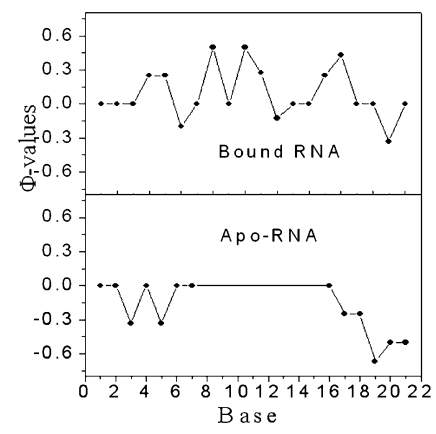

FIGURE 9. Predicted $\Phi$-values of bound RNA and apo-RNA. 


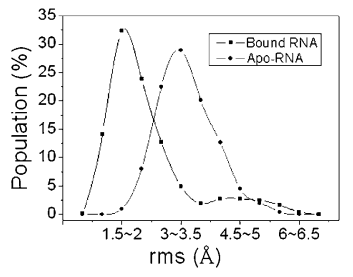

FIGURE 10. $\mathrm{C} \alpha$ RMSD conformational cluster relative to average structure.

the C $\alpha$ RMSD of bound RNA is between $1 \AA$ and $3.5 \AA$; this suggests that bound RNA has a uniform binding conformer. The conformational distribution for apo-RNA is from $1.5 \AA$ to $4.5 \AA$ and also has a single peak. Moreover, our simulation does not observe the bound conformer in apo-RNA. This suggests that bound RNA conformation is formed only after U1A binding. In summary, the folding of bound RNA is favorable that it obeys an induced-fit mechanism (Pitici et al. 2002; Turner et al. 2005; Adilakshmi et al. 2008).

\section{CONCLUSION}

Both 298 and 498K molecular dynamics simulations are performed for RNA and U1A in bound and apo states. The loop region of RNA becomes more rigid and stable upon U1A binding. This is consistent with experimental observation.

Unfolding landscape and unfolding kinetics analyses suggest that the unfolding order of bound RNA is tertiary unfolding, then U1A unbinding, and finally, RNA extension. The unfolding order of apo-RNA is tertiary unfolding, then RNA expanding. The difference of folding pathways between bound RNA and apo-RNA is the accelerated tertiary folding for RNA upon U1A binding. Unfolding kinetics and conformational cluster also indicate that the folding of bound RNA obeys an induced-fit mechanism.

The simulation results show that bound RNA and apoRNA unfold via a two-state process. The transition probability was used to determine the TSE for bound RNA and apo-RNA. Transition state analysis suggests that the TSE of bound RNA is more native-like than that of apo-RNA. The predicted $\Phi$-values suggest that U8, C10, A11, and G16 are key bases for bound RNA folding. Furthermore, this method could be used in other studies on biomolecule folding upon receptor binding.

\section{MATERIALS AND METHODS}

\section{Room-temperature and high-temperature molecular dynamics simulations}

The atomic coordinates of the snRNA-U1A complex were obtained from the crystal structure (pdb code: 1URN) (Oubridge et al. 1994). Hydrogen atoms were added using the LEAP module of AMBER8 (Case et al. 2004). Counter-ions were used to maintain system neutrality. All systems were solvated in a trun- cated octahedron box of TIP3P (Jorgensen et al. 1983) water with a buffer of $10 \AA$ A Particle mesh Ewald (PME)(Darden et al. 1993) was employed to compute long-range electrostatic interactions with the default setting in AMBER8 (Case et al. 2004). A parm99 force field was used for intramolecular interactions (Wang et al. 2000; Lwin and Luo 2006). The SHAKE algorithm (Rychaert et al. 1977) was used to constrain the bonds involving hydrogen atoms. A 1000-step steepest descent minimization was performed to relieve any structural clash in the solvated systems. Then 20-ps simulation was performed by heating up and performing brief equilibration in the NVT ensemble at $298 \mathrm{~K}$. Langevin dynamics with a time step of $2 \mathrm{fs}$ was used in the heating and equilibration runs with a friction constant of $1 \mathrm{ps}^{-1}$.

To study the folded state of each solvated system, 10 independent trajectories, $10.0 \mathrm{~ns}$ each, in the NPT ensemble (Berendsen et al. 1984) at $298 \mathrm{~K}$ were then simulated with PMEMD of AMBER8. To investigate the unfolding pathway of each solvated system, 10 independent trajectories of $20 \mathrm{~ns}$ each were performed for the RNA-U1A complexes in the NVT ensemble at $498 \mathrm{~K}$. Ten independent trajectories, $10 \mathrm{~ns}$ each, were simulated for the apo-RNA and apo-U1A systems. To confirm the influence of a mutant for Arg52Gln, a 10-ns simulation was performed for the RNA-mutant U1A at 298 and 498K. A total of 720-ns trajectories were collected for four systems (the RNA-U1A complex, apo-RNA, apo-U1A, and RNA-mutant U1A) at both 298 and $498 \mathrm{~K}$, taking about $84,200 \mathrm{CPU}$ hours on the xeon $(3.0$ $\mathrm{GHz}$ ) cluster.

Native contacts between RNA and U1A were monitored to detect the beginning of the unfolded state. It was found that $15 \mathrm{~ns}$ at $498 \mathrm{~K}$ were needed to reach the equilibrium stage for the complex, so that the first $15 \mathrm{~ns}$ (a total of $150 \mathrm{~ns}$ for the complex) were used to study the unfolding kinetics, and the remaining $5 \mathrm{~ns}$ (a total of $50 \mathrm{~ns}$ for the complex) were used to monitor the unfolded state. Native contacts within apo-RNA itself were also monitored to detect the beginning of its unfolded state, so the first 8 ns were used to study the unfolding kinetics and the remaining 2 ns (a total of $20 \mathrm{~ns}$ for apo-RNA) were used to monitor the unfolded state.

\section{Transition state simulations}

According to the definition of the transition state (TS), 40 test MD runs for each candidate snapshot were performed to calculate the transition probability $(P)$ (Pande and Rokhsar 1999; Gsponer and Caflisch 2002; Chong et al. 2005). All simulations had the same initial conformation, but differing initial velocities. TS simulations were done at $498 \mathrm{~K}$ to accelerate the simulated folding/unfolding rate. Each test trajectory was terminated when the conformation had reached the folded or unfolded state. The folded state is defined as the $\mathrm{C}^{\prime}{ }^{\prime} \mathrm{RMSD}$ within $9.0 \AA$ from the average structure of the folded state at $298 \mathrm{~K}$. Up to a 1-ns simulation at $498 \mathrm{~K}$ was found to be sufficient for each test trajectory (Chen and Luo 2007; Chen 2008; Qin et al. 2009), i.e., $P$-values for tested snapshots were no longer changing when longer simulations were done.

\section{Data analysis}

Tertiary contact assignment was handled with in-house software (Chen and Luo 2007; Chen 2008, 2009a,b; Qin et al. 2009). These 
residues and nucleotides are in contact when their side chains are closer than $6.5 \AA$ for the complex. The nonadjacent nucleotides are in contact when their bases are closer than $7.5 \AA$ within RNA. Electrostatic interactions are assigned when the distance between the positive charge residue and the RNA phosphate backbone is less than $11 \AA$ (Garcia-Garcia and Draper 2003). Secondary structure assignment was performed with DSSP (Kabsch and Sander 1983). RNA sugar conformation of C3'-endo and C2'endo was mapped with $\mathrm{R}$ language. The unfolding kinetics was fitted in Origin 7.0. The unfolding landscape was performed by calculating the normalized probability from histogram analysis (Pande and Rokhsar 1999). Here we used the fraction of native tertiary contacts $\left(Q_{f}\right)$, the fraction of native binding contacts $\left(Q_{b}\right)$, and the radius of gyration $\left(R_{g}\right)$ to map the unfolding landscapes. Representative structures at unfolding half-times were used to construct the unfolding pathways.

According to the definition of protein $\Phi$-values, which were computed with a strategy similar to those used in other studies (Caflisch and Karplus 1994; Vendruscolo et al. 2001; Gsponer and Caflisch 2002), this method might be suitable for the calculation of RNA $\Phi$-values. Therefore, the following equation was used to process the RNA $\Phi$-values:

$$
\Phi_{\mathrm{RNAi}}{ }^{\mathrm{calc}}=\frac{N_{i}^{\mathrm{TS}}-N_{i}^{\mathrm{U}}}{N_{i}^{\mathrm{F}}-N_{i}^{\mathrm{U}}},
$$

where $N_{i}^{\mathrm{TS}}$ is the number of native contacts for base $i$ at the transition state, and $N_{i}^{\mathrm{F}}$ and $N_{i}^{\mathrm{U}}$ are the number of native contacts for base $i$ at the folded and unfolded states, respectively.

\section{SUPPLEMENTAL MATERIAL}

Supplemental material can be found at http://www.rnajournal.org.

\section{ACKNOWLEDGMENTS}

This work was supported by the Instrumental Analysis Center of Shanghai Jiaotong University; by the National Natural Science Foundation of China (Grant Nos. 30770502 and 20773085); by the Natural Science Foundation of Shanghai China (Grant No. 10ZR1414500); in part by grants from Ministry of Science and Technology China (2010CB833601); and by the National 863 High-Tech Program (2007DFA31040).

Received November 15, 2009; accepted February 7, 2010.

\section{REFERENCES}

Adilakshmi T, Bellur DL, Woodson SA. 2008. Concurrent nucleation of $16 \mathrm{~S}$ folding and induced fit in $30 \mathrm{~S}$ ribosome assembly. Nature 455: $1268-1272$.

Baker D. 1998. Metastable states and folding free energy barriers. Nat Struct Biol 5: 1021-1024.

Berendsen HJC, Postma JPM, van Gunsteren WF, DiNola A, Haak JR. 1984. Molecular dynamics with coupling to an external bath. J Chem Phys 81: 3684-3690.

Blakaj DM, McConnell KJ, Beveridge DL, Baranger AM. 2001. Molecular dynamics and thermodynamics of protein-RNA interactions: Mutation of a conserved aromatic residue modifies stacking interactions and structural adaptation in the U1A-stemloop 2 RNA complex. J Am Chem Soc 123: 2548-2551.
Boehr DD, Wright PE. 2008. Biochemistry. How do proteins interact? Science 320: 1429-1430.

Bowman GR, Huang X, Yao Y, Sun J, Carlsson G, Guibas LJ, Pande VS. 2008. Structural insight into RNA hairpin folding intermediates. J Am Chem Soc 130: 9676-9678.

Caflisch A, Karplus M. 1994. Molecular-dynamics simulation of protein denaturation-solvation of the hydrophobic cores and secondary structure of barnase. Proc Natl Acad Sci 91: 1746-1750.

Case DA, Darden TA, Cheatham TE, Simmerling CL III, Wang J, Duke RE, Luo R, Merz KM, Wang B, Pearlman DA, et al. 2004. $A M B E R$ 8. University of California, San Francisco.

Chen HF. 2008. Mechanism of coupled folding and binding in the siRNA-PAZ complex. J Chem Theory Comput 4: 1360-1368.

Chen HF. 2009a. Aggregation mechanism investigation of the GIFQINS cross- $\beta$ amyloid fibril. Comput Biol Chem 33: 41-45.

Chen HF. 2009b. Molecular dynamics simulation of phosphorylated KID post-translational modification. PLoS One 4: e6516. doi: 10.1371/journal.pone.0006516.

Chen HF, Luo R. 2007. Binding induced folding in p53-MDM2 complex. J Am Chem Soc 129: 2930-2937.

Chong LT, Snow CD, Rhee YM, Pande VS. 2005. Dimerization of the p53 oligomerization domain: Identification of a folding nucleus by molecular dynamics simulations. J Mol Biol 345: 869-878.

Darden T, York D, Pedersen L. 1993. Particle mesh Ewald: An N $\log (N)$ method for Ewald sums in large systems. J Chem Phys 98: 10089-10092.

Day R, Daggett V. 2005. Ensemble versus single-molecule protein unfolding. Proc Natl Acad Sci 102: 13445-13450.

Day R, Daggett V. 2007. Direct observation of microscopic reversibility in single-molecule protein folding. J Mol Biol 366: 677-686.

Fernandez-Escamilla AM, Cheung MS, Vega MC, Wilmanns M, Onuchic JN, Serrano L. 2004. Solvation in protein folding analysis: Combination of theoretical and experimental approaches. Proc Natl Acad Sci 101: 2834-2839.

Fersht AR. 2000. Transition-state structure as a unifying basis in protein-folding mechanisms: Contact order, chain topology, stability, and the extended nucleus mechanism. Proc Natl Acad Sci 97: 1525-1529.

Fersht AR, Daggett V. 2002. Protein folding and unfolding at atomic resolution. Cell 108: 573-582.

Fersht AR, Matouschek A, Serrano L. 1992. The folding of an enzyme. I. Theory of protein engineering analysis of stability and pathway of protein folding. J Mol Biol 224: 771-782.

Garcia-Garcia C, Draper DE. 2003. Electrostatic interactions in a peptide-RNA complex. J Mol Biol 331: 75-88.

Green MR. 1991. Biochemical mechanisms of constitutive and regulated pre-mRNA splicing. Annu Rev Cell Biol 7: 559-599.

Gsponer J, Caflisch A. 2002. Molecular dynamics simulations of protein folding from the transition state. Proc Natl Acad Sci 99: 6719-6724.

Hall KB. 1994. Interaction of RNA hairpins with the human U1A N-terminal RNA binding domain. Biochemistry 33: 10076-10088.

Hermann T, Westhof E. 1999. Simulations of the dynamics at an RNA-protein interface. Nat Struct Biol 6: 540-544.

Hyeon C, Thirumalai D. 2005. Mechanical unfolding of RNA hairpins. Proc Natl Acad Sci 102: 6789-6794.

James LC, Tawfik DS. 2003. Conformational diversity and protein evolution-a 60-year-old hypothesis revisited. Trends Biochem Sci 28: $361-368$.

Jorgensen WL, Chandrasekhar J, Madura JD, Impey RW, Klein ML. 1983. Comparison of simple potential functions for simulating liquid water. J Chem Phys 79: 926-935.

Kabsch W, Sander C. 1983. Dictionary of protein secondary structure: Pattern recognition of hydrogen-bonded and geometrical features. Biopolymers 22: 2577-2637.

Kormos BL, Baranger AM, Beveridge DL. 2006. Do collective atomic fluctuations account for cooperative effects? Molecular dynamics studies of the U1A-RNA complex. J Am Chem Soc 128: 89928993. 
Kormos BL, Baranger AM, Beveridge DL. 2007. A study of collective atomic fluctuations and cooperativity in the U1A-RNA complex based on molecular dynamics simulations. J Struct Biol 157: 500513.

Koshland DE. 1958. Application of a theory of enzyme specificity to protein synthesis. Proc Natl Acad Sci 44: 98-104.

Kumar S, Ma B, Tsai CJ, Sinha N, Nussinov R. 2000. Folding and binding cascades: Dynamic landscapes and population shifts. Protein Sci 9: 10-19.

Lange OF, Lakomek NA, Fares C, Schroder GF, Walter KF, Becker S, Meiler J, Grubmuller H, Griesinger C, de Groot BL. 2008. Recognition dynamics up to microseconds revealed from an RDC-derived ubiquitin ensemble in solution. Science 320: 14711475.

Law MJ, Linde ME, Chambers EJ, Oubridge C, Katsamba PS, Nilsson L, Haworth IS, Laird-Offringa IA. 2006. The role of positively charged amino acids and electrostatic interactions in the complex of U1A protein and U1 hairpin II RNA. Nucleic Acids Res 34: $275-285$.

Lu J, Hall KB. 1995. An RBD that does not bind RNA: NMR secondary structure determination and biochemical properties of the C-terminal RNA binding domain from the human U1A protein. J Mol Biol 247: 739-752.

Lührmann R, Kastner B, Bach M. 1990. Structure of spliceosomal snRNPs and their role in pre-mRNA splicing. Biochim Biophys Acta 1087: 265-292.

Lwin TZ, Luo R. 2006. Force field influences in $\beta$-hairpin folding simulations. Protein Sci 15: 2642-2655.

Ma B, Kumar S, Tsai CJ, Nussinov R. 1999. Folding funnels and binding mechanisms. Protein Eng 12: 713-720.

Ma B, Shatsky M, Wolfson HJ, Nussinov R. 2002. Multiple diverse ligands binding at a single protein site: A matter of pre-existing populations. Protein Sci 11: 184-197.

McCully ME, Beck DA, Daggett V. 2008. Microscopic reversibility of protein folding in molecular dynamics simulations of the engrailed homeodomain. Biochemistry 47: 7079-7089.

Murray LJ, Arendall WB 3rd, Richardson DC, Richardson JS. 2003. RNA backbone is rotameric. Proc Natl Acad Sci 100: 1390413909.

Nagai K, Oubridge C, Jessen TH, Li J, Evans PR. 1990. Crystal structure of the RNA-binding domain of the U1 small nuclear ribonucleoprotein A. Nature 348: 515-520.

Okazaki K, Takada S. 2008. Dynamic energy landscape view of coupled binding and protein conformational change: Induced-fit versus population-shift mechanisms. Proc Natl Acad Sci 105: 11182-11187.

Onoa B, Tinoco I Jr. 2004. RNA folding and unfolding. Curr Opin Struct Biol 14: 374-379.

Oubridge C, Ito N, Evans PR, Teo CH, Nagai K. 1994. Crystal structure at 1.92 A resolution of the RNA-binding domain of the U1A spliceosomal protein complexed with an RNA hairpin. Nature 372: 432-438.

Pande VS, Rokhsar DS. 1999. Molecular dynamics simulations of unfolding and refolding of a $\beta$-hairpin fragment of protein G. Proc Natl Acad Sci 96: 9062-9067.
Pitici F, Beveridge DL, Baranger AM. 2002. Molecular dynamics simulation studies of induced fit and conformational capture in U1A-RNA binding: Do molecular substates code for specificity? Biopolymers 65: 424-435.

Qin F, Chen Y, Li YX, Chen HF. 2009. Induced fit of mRNA-TIS11D complex. J Chem Phys 131: 115103. doi: 10.1063/1.3224126.

Reyes CM, Kollman PA. 1999. Molecular dynamics studies of U1ARNA complexes. RNA 5: 235-244.

Rychaert JP, Ciccotti G, Berendsen HJC. 1977. Numerical integration of Cartesian equations of motion of a system with constraints: Molecular dynamics of n-alkanes. Comput Phys 23: 327-341.

Sato S, Fersht AR. 2007. Searching for multiple folding pathways of a nearly symmetrical protein: Temperature dependent $\varphi$-value analysis of the B domain of protein A. J Mol Biol 372: 254-267.

Scherly D, Boelens W, van Venrooij WJ, Dathan NA, Hamm J, Mattaj IW. 1989. Identification of the RNA binding segment of human U1 A protein and definition of its binding site on U1 snRNA. EMBO J 8: 4163-4170.

Shea JE, Brooks CL 3rd. 2001. From folding theories to folding proteins: A review and assessment of simulation studies of protein folding and unfolding. Annu Rev Phys Chem 52: 499-535.

Showalter SA, Hall KB. 2005. Correlated motions in the U1 snRNA stem-loop 2:U1A RBD1 complex. Biophys J 89: 2046-2058.

Tang Y, Nilsson L. 1999. Molecular dynamics simulations of the complex between human U1A protein and hairpin II of U1 small nuclear RNA and of free RNA in solution. Biophys J 77: 12841305.

Tsai CJ, Ma B, Nussinov R. 1999. Folding and binding cascades: Shifts in energy landscapes. Proc Natl Acad Sci 96: 9970-9972.

Tsai CJ, Ma B, Sham YY, Kumar S, Nussinov R. 2001. Structured disorder and conformational selection. Proteins 44: 418-427.

Turjanski AG, Gutkind JS, Best RB, Hummer G. 2008. Bindinginduced folding of a natively unstructured transcription factor. PLoS Comput Biol 4: e1000060. doi: 10.1371/journal.pcbi.1000060.

Turner B, Melcher SE, Wilson TJ, Norman DG, Lilley DM. 2005. Induced fit of RNA on binding the L7Ae protein to the kink-turn motif. RNA 11: 1192-1200.

van Gelder CW, Gunderson SI, Jansen EJ, Boelens WC, PolycarpouSchwarz M, Mattaj IW, van Venrooij WJ. 1993. A complex secondary structure in U1A pre-mRNA that binds two molecules of U1A protein is required for regulation of polyadenylation. EMBO J 12: 5191-5200.

Vendruscolo M, Paci E, Dobson CM, Karplus M. 2001. Three key residues form a critical contact network in a protein folding transition state. Nature 409: 641-645.

Wang JM, Cieplak P, Kollman PA. 2000. How well does a restrained electrostatic potential (RESP) model perform in calculating conformational energies of organic and biological molecules? J Comput Chem 21: 1049-1074.

Weikl TR, von Deuster C. 2009. Selected-fit versus induced-fit protein binding: Kinetic differences and mutational analysis. Proteins 75: 104-110.

Zhao Y, Kormos BL, Beveridge DL, Baranger AM. 2006. Molecular dynamics simulation studies of a protein-RNA complex with a selectively modified binding interface. Biopolymers 81: 256-269. 

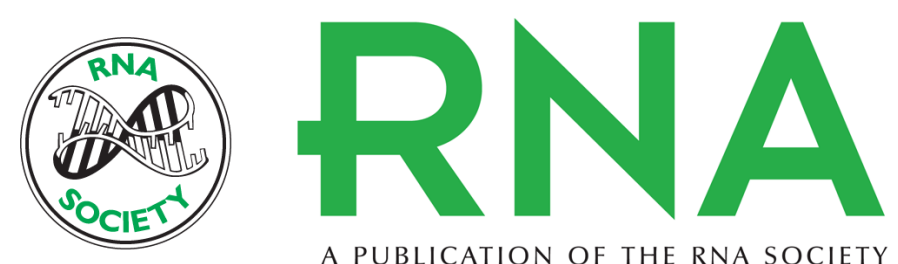

A PUBLICATION OF THE RNA SOCIETY

\section{Induced fit or conformational selection for RNA/U1A folding}

Fang Qin, Yue Chen, Maoying Wu, et al.

RNA 2010 16: 1053-1061 originally published online March 30, 2010

Access the most recent version at doi:10.1261/rna.2008110

Supplemental

Material

References

\section{License}

Email Alerting Service
http://rnajournal.cshlp.org/content/suppl/2010/03/23/rna.2008110.DC1

This article cites 64 articles, 15 of which can be accessed free at: http://rnajournal.cshlp.org/content/16/5/1053.full.html\#ref-list-1 